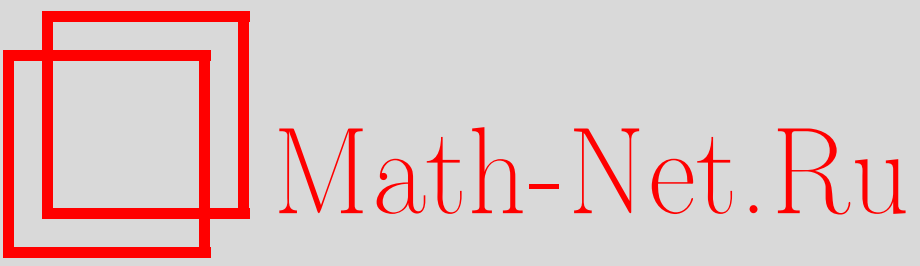

Г. М. Фельдман, Гауссовские распределения в смысле Бернштейна: факторизация, носители, закон нуля или единицы, Теория вероятн. и ее примен., 2011, том 56, выпуск 3, 494-513

DOI: https://doi.org/10.4213/tvp4404

Использование Общероссийского математического портала Math-Net.Ru подразумевает, что вы прочитали и согласны с пользовательским соглашением

http: //www . mathnet.ru/rus/agreement

Параметры загрузки:

IP : 54.198 .187 .58

26 апреля 2023 г., 12:43:01 
ТЕОРИЯ ВЕРОЯТНОСТЕЙ

Том 56

И ЕЕ ПРИМЕНЕНИЯ

Выпуск 3

2011

(c) 2011 г.

ФЕЛЬДМАН Г. М.*

\section{ГАУССОВСКИЕ РАСПРЕДЕЛЕНИЯ \\ В СМЫСЛЕ БЕРНШТЕЙНА: ФАКТОРИЗАЦИЯ, НОСИТЕЛИ, ЗАКОН НУЛЯ ИЛИ ЕДИНИЦЫ}

Пусть $X$ - локально компактная абелева группа, удовлетворяющая второй аксиоме счетности, $\mu$ - гауссовское распределение в смысле Бернштейна на $X$. В предположении, что связная компонента нуля группы $X$ содержит конечное число элементов порядка 2 , доказано, что $\mu$ представимо в виде свертки гауссовского распределения, распределения Хаара компактной подгруппы $X$ и некоторого заряда. Описаны носители $\mu$ на произвольной группе $X$. Для группы $X$, компонента нуля которой имеет конечную размерность, доказан закон нуля или единицы для $\mu$ при условии, что $\mu$ не имеет идемпотентных делителей.

Ключевые слова и фразы: локально компактная абелева группа, теорема Каца-Бернштейна, гауссовское распределение, закон нуля или единицы.

1. Введение. Согласно классической теореме Каца-Бернштейна, если $\xi_{1}, \xi_{2}$ - независимые случайные величины и сумма $\xi_{1}+\xi_{2}$ и разность $\xi_{1}-\xi_{2}$ независимы, то случайные величины $\xi_{j}-$ гауссовские. Групповым аналогам этой теоремы посвящен ряд работ (см. [1]-[10], [11, гл. III]). Настоящая статья продолжает эти исследования.

Пусть $X$ - локально компактная абелева группа. Мы будем рассматривать в статье лишь группы, удовлетворяющие второй аксиоме счетности, и поэтому не будем это специально оговаривать.

Распределение $\mu$ на группе $X$ называется гауссовским распределением в смысле Бернштейна, если из того, что $\xi_{1}$ и $\xi_{2}$ - независимые одинаково распределенные случайные величины со значениями в группе $X$ и с распределением $\mu$, следует, что их сумма и разность независимы. Таким образом, из теоремы Каца-Бернштейна вытекает, что на вещественной прямой класс гауссовских распределений совпадает с классом гауссовских распределений в смысле Бернштейна. В групповой ситуации, однако, это, вообще говоря, не так. Несложно доказать, что на

*Физико-технический институт низких температур им. Б. И. Веркина НАН Украины, пр-т Ленина, 47, 61103, Харьков, Украина; e-mail: feldman@ilt.kharkov.ua 
произвольной локально компактной абелевой группе $X$ к классу гауссовских распределений в смысле Бернштейна принадлежат гауссовские распределения, распределения Хаара некоторых компактных подгрупп группы $X$, а также их свертки.

В работах А. Л. Рухина [1], [2] и Х. Хейера и Ч. Ролла [3] были получены некоторые достаточные условия, которым должна удовлетворять группа $X$, для того чтобы любое гауссовское распределение в смысле Бернштейна $\mu$ на группе $X$ являлось сверткой гауссовского распределения и некоторого распределения Хаара компактной подгруппы группы $X$. Это означает, что $\mu$ инвариантно относительно некоторой компактной подгруппы $K$ группы $X$ и $\mu$ при естественном гомоморфизме $X \rightarrow X / K$ индуцирует на факторгруппе $X / K$ гауссовское распределение. Полное описание таких групп $X$ было дано в работах [4] и [9]. Это группы, связная компонента нуля которых содержит не более одного элемента порядка 2.

В п. 2 мы доказываем, что если связная компонента нуля группы $X$ содержит конечное число элементов порядка 2 , то любое гауссовское распределением в смысле Бернштейна на группе $X$ является сверткой гауссовского распределения, распределения Хаара компактной подгруппы группы $X$ и заряда с носителем в подгруппе, порожденной элементами порядка 2 группы $X$.

В п. 3 мы описываем носители гауссовских распределений в смысле Бернштейна на произвольной группе $X$.

Закон нуля или единицы для гауссовских распределений в смысле Бернштейна на топологических абелевых группах (не обязательно локально компактных) был подробно изучен в работах Т. Бычковского [12], [13] и Г. Бычковской и Т. Бычковского [14]. В частности, в [14] доказано, что если $G$ - топологическая абелева группа, не содержащая элементов порядка 2 , а $\mu$ - гауссовское распределение в смысле Бернштейна на $G$, не имеющее идемпотентных делителей, то для $\mu$ имеет место закон нуля или единицы, т.е. для любой борелевской подгруппы $K \subset G$ либо $\mu(K)=0$, либо $\mu(K)=1$. В п. 4 мы доказываем, что если $X-$ локально компактная абелева группа такая, что ее связная компонента нуля имеет конечную размерность, то на группе $X$ справедлив закон нуля или единицы для гауссовских распределений в смысле Бернштейна, не имеющих идемпотентных делителей.

Нам понадобятся некоторые результаты, относящиеся к структуре локально компактных абелевых групп и теории двойственности (см. [15, гл. 6]). Условимся об обозначениях. Обозначим через $Y=X^{*}$ группу характеров группы $X$, а через $(x, y)$ - значение характера $y \in Y$ на элементе $x \in X$. Если $K$ - замкнутая подгруппа $X$, то $A(Y, K)=\{y \in$ $Y:(x, y)=1$ для всех $x \in K\}$ обозначает ее аннулятор. Обозначим через $c_{X}$ связную компоненту нуля группы $X$, а через $b_{Y}$ - подгруппу в $Y$, 
состоящую из всех компактных элементов группы $Y$. Если $A$ и $B-$ подмножества в $X$, то положим $A+B=\{x \in X: x=a+b, a \in A$, $y \in B\}$. Обозначим через $\mathbf{R}$ группу вещественных чисел, через $\mathbf{T}-$ группу вращений окружности (одномерный тор), т.е. $\mathbf{T}=\{z \in \mathbf{C}:|z|=$ $1\}$, через $\mathbf{Z}$ - группу целых чисел. Обозначим через $\mathbf{Z}(n)$ циклическую группу, состоящую из $n$ элементов. Мы будем представлять $\mathbf{Z}(n)$ как группу корней степени $n$ из единицы. Обозначим $X_{(n)}=\{x \in X: n x=$ $0\}, X^{(n)}=\{x \in X: x=n \widetilde{x}, \widetilde{x} \in X\}$. Группа $X$ называется группой Корвина, если $X^{(2)}=X$.

Сверточную полугруппу вероятностных распределений на $X$ обозначим через $M^{1}(X)$. Через $E_{x}$ обозначим вырожденное распределение, сосредоточенное в точке $x \in X$. Для $\mu \in M^{1}(X)$ обозначим через $\sigma(\mu)$ носитель $\mu$. Характеристическую функцию распределения $\mu \in M^{1}(X)$ определим формулой

$$
\widehat{\mu}(y)=\int_{X}(x, y) d \mu(x) .
$$

Распределение $\gamma \in M^{1}(X)$ называется гауссовским (см. [16]), если его характеристическая функция представима в виде

$$
\widehat{\gamma}(y)=(x, y) \exp \{-\varphi(y)\}, \quad y \in Y,
$$

где $x \in X$, а $\varphi(y)$ - непрерывная неотрицательная функция на $Y$, удовлетворяющая уравнению

$$
\varphi(u+v)+\varphi(u-v)=2[\varphi(u)+\varphi(v)], \quad u, v \in Y .
$$

Пусть $\Gamma(X)$ обозначает множество гауссовских распределений на группе $X$. Гауссовское распределение $\gamma$ называется симметричным, если в (1) $x=0$. Множество симметричных гауссовских распределений на группе $X$ обозначим через $\Gamma^{\mathrm{s}}(X)$. Как доказано в [16], носитель симметричного гауссовского распределения - некоторая связная подгруппа в $X$. Отметим, что любая связная локально компактная абелева группа топологически изоморфна группе вида $\mathbf{R}^{l} \times K$, где $l \geqslant 0$, а $K-$ связная компактная абелева группа. Класс связных компактных абелевых групп достаточно обширен. Именно, каждая такая группа топологически изоморфна группе характеров некоторой дискретной абелевой группы без кручения. Хорошая структурная теория для дискретных абелевых групп без кручения в случае, когда ранг группы больше 1 , отсутствует (см., например, $[17, \S 93])$. А значит, такая теория отсутствует и для связных компактных абелевых групп размерности больше 1.

Пусть $\Gamma_{\mathrm{B}}(X)$ обозначает множество гауссовских распределений в смысле Бернштейна на группе $X$. Обозначим через $m_{X}$ меру Хаapa на $X$. Если группа $X$ компактна, мы будем предполагать, что 
$m_{X} \in M^{1}(X)$. Пусть $K-$ компактная подгруппа группы $X$. Тогда характеристическая функция распределения Хаара $m_{K}$ имеет вид

$$
\widehat{m}_{K}(y)= \begin{cases}1, & y \in A(Y, K), \\ 0, & y \notin A(Y, K) .\end{cases}
$$

2. Факторизация гауссовских распределений в смысле Бернштейна. Пусть $\xi_{1}$ и $\xi_{2}-$ независимые одинаково распределенные случайные величины со значениями в группе $X$, имеющие распределение $\mu$. Нетрудно проверить, что сумма $\xi_{1}+\xi_{2}$ и разность $\xi_{1}-\xi_{2}$ независимы, т.е. $\mu \in \Gamma_{\mathrm{B}}(X)$, в том и только том случае, когда характеристическая функция $\widehat{\mu}(y)=\mathbf{E}\left[\left(\xi_{j}, y\right)\right]$ удовлетворяет уравнению

$$
\widehat{\mu}(u+v) \widehat{\mu}(u-v)=\widehat{\mu}^{2}(u)|\widehat{\mu}(v)|^{2}, \quad u, v \in Y .
$$

Основным результатом этого пункта является следующая теорема.

Теорема 1. Пусть $X-$ локально компактная абелева группа. Предположим, что связная компонента нуля группы $X$ содержит конечное число әлементов порядка 2 . Если $\mu \in \Gamma_{\mathrm{B}}(X)$, mо

$$
\mu=\gamma * m_{K} * \delta
$$

где $\gamma \in \Gamma(X), K-$ компактная подгруппа Корвина, а $\delta-$ зарядн на $X_{(2)}$ maкой, чmo $\delta^{* 2}=E_{0}$.

Для доказательства теоремы 1 нам понадобятся следующие леммы.

Лемма 1 (см. [4]). Пусть $X-$ локально компактная абелева группа. Пусть $\mu \in \Gamma_{\mathrm{B}}(X)$. Тогда носитель $\sigma(\mu)$ содержится в классе смежности некоторой подгруппь в $X$, топологически изомор $\phi$ ной группе вида

$$
\mathbf{R}^{l} \times K
$$

где $l \geqslant 0$, а $K-$ компактная группа Корвина.

Лемма 2. Пусть локально компактная абелева группа $X$ имеет вид (5). Тогда $\overline{Y^{(2)}}=Y^{(2)}$ u $X_{(2)} \subset c_{X}$.

Д ок аз а т ель с т в о. Мы имеем $Y \cong \mathbf{R}^{l} \times L$, где группа $L=$ $K^{*}$ - дискретна. Чтобы не усложнять обозначения, мы будем предполагать, что $Y=\mathbf{R}^{l} \times L$. Очевидно, что $\overline{Y^{(2)}}=Y^{(2)}$. Из $K^{(2)}=K$ следует, что $L_{(2)}=\{0\}$, т.е. группа $L$ не содержит элементов порядка 2. Отсюда следует, что подгруппа $b_{L}$ состоит из нуля и множества всех элементов нечетного порядка группы $L$. Следовательно, $\left(b_{L}\right)^{(2)}=b_{L}$, a значит, $b_{L} \subset Y^{(2)}$. Поскольку очевидно, что $b_{Y}=b_{L}$, мы имеем $c_{X}=A\left(X, b_{Y}\right)=A\left(X, b_{L}\right) \supset A\left(X, Y^{(2)}\right)=X_{(2)}$. Лемма доказана. 
Лемма 3 (см. [4]). Пусть $X-$ локально компактная абелева группа. Пусть $\mu \in \Gamma_{\mathrm{B}}(X)$, причем характеристическая функиия $\widehat{\mu}(y)$ не обращается в нуль. Тогда характеристическая функция $\widehat{\mu}(y)$ представима в виде

$$
\widehat{\mu}(y)=l(y) \exp \{-\varphi(y)\}, \quad y \in Y,
$$

где функция $l(y)$ удовлетворяет уравнению

$$
l(u+v) l(u-v)=l^{2}(u), \quad u, v \in Y,
$$

и условиям: $l(y)$ непрерывна, $l(-y)=\overline{l(y)},|l(y)|=1, l(0)=1, a \varphi(y)-$ непрерывная неотрицательная функиия на $Y$, удовлетворяющая уравнению (2).

Лемма 4. Пусть $X-$ конечная абелева группа такая, что все ее ненулевые элементы имеют порядок 2. Тогда для любой вещественнозначной функиии $f(y)$ на группе $Y$ существует заряд $\delta$ на $X$ такой, uто $\widehat{\delta}(y)=f(y), y \in Y$.

Д ок аз а т ель с т в о. Пусть $X=\left\{x_{1}, \ldots, x_{n}\right\}, Y=\left\{y_{1}, \ldots, y_{n}\right\}$, $\delta$ - комплекснозначная мера на $X$ и $\delta\left(\left\{x_{j}\right\}\right)=a_{j}, j=1, \ldots, n$. Тогда характеристическая функция $\widehat{\delta}(y)$ имеет вид

$$
\widehat{\delta}\left(y_{i}\right)=\sum_{j=1}^{n} a_{j}\left(x_{j}, y_{i}\right), \quad i=1, \ldots, n .
$$

Если числа $\widehat{\delta}\left(y_{i}\right)=f\left(y_{i}\right)$ заданы, то мы имеем систему линейных уравнений для нахождения $a_{j}$. Эта система всегда имеет решение, так как ее определитель $\operatorname{det}\left(x_{j}, y_{i}\right)$ не равен 0. Таким образом, существование комплекснозначной меры $\delta$ доказано.

Если все отличные от нуля элементы группы $X$ имеют порядок 2 , то мы имеем $\left(x_{j}, y_{i}\right)= \pm 1$. Поскольку функция $f(y)$ вещественнозначная, отсюда следует, что все числа $a_{j}$ вещественны, т.е. $\delta$ - заряд. Лемма доказана.

Док а з а т ель с т в о т е о р емы 1 . Пусть $\mu \in \Gamma_{B}(X)$. По лемме $1 \sigma(\mu)$ содержится в классе смежности группы $X$ относительно некоторой подгруппы $G \subset X$, топологически изоморфной группе вида (5). Поэтому, заменяя, если это необходимо, распределение $\mu$ его сдвигом, будем предполагать с самого начала, что группа $X$ имеет вид (5). Тогда по лемме 2 имеем $X_{(2)} \subset c_{X}$, а значит, подгруппа $X_{(2)}$ конечна. Кроме того, $\overline{Y^{(2)}}=Y^{(2)}$. Очевидно, что $A\left(Y, X_{(2)}\right)=\overline{Y^{(2)}}=Y^{(2)}$. Предположим, что число элементов подгруппы $X_{(2)}$ равно $n$. Поскольку $X_{(2)} \cong\left(X_{(2)}\right)^{*} \cong Y / A\left(Y, X_{(2)}\right)=Y / Y^{(2)}$, то разложение группы $Y$ по подгруппе $Y^{(2)}$ имеет вид

$$
Y=\bigcup_{j=0}^{n-1}\left(y_{j}+Y^{(2)}\right), \quad y_{0}=0 .
$$


Рассмотрим множество $E=\{y \in Y: \widehat{\mu}(y) \neq 0\}$. Из (4) вытекает, что $E$ - открытая подгруппа в $Y$, удовлетворяющая условию $E \cap Y^{(2)}=E^{(2)}$. Пусть $H$ - обозначает объединение тех классов смежности в (8), для которых $E \cap\left(y_{j}+Y^{(2)}\right) \neq \varnothing$. Изменяя, если это необходимо, нумерацию элементов $y_{j}$, можно считать, что

$$
H=\bigcup_{j=0}^{l-1}\left(y_{j}+Y^{(2)}\right), \quad y_{j} \in E, \quad j=0,1, \ldots, l-1 .
$$

Так как $E$ - подгруппа в $Y$, то и $H-$ подгруппа в $Y$. Легко видеть, что $\overline{E^{(2)}}=E^{(2)}$ и разложение группы $E$ по подгруппе $E^{(2)}$ имеет вид

$$
E=\bigcup_{j=0}^{l-1}\left(y_{j}+E^{(2)}\right)
$$

По лемме 3 ограничение характеристической функции $\widehat{\mu}(y)$ на $E$ допускает представление (6). Меняя в (7) $u$ и $v$ местами, получаем

$$
l(u+v) l(v-u)=l^{2}(v), \quad u, v \in E .
$$

Перемножая (7) и (10), находим

$$
l^{2}(u+v)=l^{2}(u) l^{2}(v), \quad u, v \in E,
$$

т.е. функция $l^{2}(y)-$ характер группы $E$. С другой стороны, полагая в (7) $u=v=y$, получаем

$$
l(2 y)=l^{2}(y), \quad y \in E .
$$

Из (11) и (12) следует, что сужение функции $l(y)$ на $E^{(2)}$ - характер группы $E^{(2)}$. Продолжим этот характер с $E^{(2)}$ до характера группы $Y$. По теореме двойственности существует такой элемент $x_{0} \in X$, что $l(y)=\left(x_{0}, y\right), y \in E^{(2)}$. Рассмотрим функцию $l^{\prime}(y)=\left(-x_{0}, y\right) l(y), y \in E$. Эта функция удовлетворяет уравнению (7), а также всем условиям, которым удовлетворяет функция $l(y)$. Кроме того, $l^{\prime}(2 y)=1, y \in E$. Из (12) следует тогда, что $l^{\prime}(y)= \pm 1, y \in E$. Поэтому из (10) получаем $l^{\prime}(u+v) l^{\prime}(u-v)=1, u, v \in E$, а значит, $l^{\prime}(u+v)=l^{\prime}(u-v), u, v \in E$. Таким образом, $l^{\prime}(u+2 v)=l^{\prime}(u), u, v \in E$. Поскольку функция $l^{\prime}(y)$ постоянна на классах смежности группы $E$ по подгруппе $E^{(2)}$, то из (8) и (9) следует, что функцию $l^{\prime}(y)$ можно продолжить с $E$ на $Y$, полагая $\widetilde{l}^{\prime}\left(y_{j}+u\right)=l^{\prime}\left(y_{j}\right), u \in Y^{(2)}, j=0,1, \ldots, l-1$, и $\widetilde{l}^{\prime}\left(y_{j}+u\right)=1, u \in Y^{(2)}$, $j=l, l+1, \ldots, n-1$. Продолженная функция $\widetilde{l}^{\prime}(y)$ инвариантна относительно подгруппы $Y^{(2)}$, а значит, определяет некоторую функцию на 
факторгруппе $Y / Y^{(2)}$. Так как $\left(X_{(2)}\right)^{*} \cong Y / Y^{(2)}$, то по лемме 4 существует такой заряд $\delta$ на $X_{(2)}$, что $\widehat{\delta}(y)=\widetilde{l}^{\prime}(y), y \in Y$. В результате мы получаем следующее представление:

$$
\widehat{\mu}(y)= \begin{cases}\left(x_{0}, y\right) \exp \{-\varphi(y)\} \widehat{\delta}(y), & y \in E, \\ 0, & y \notin E .\end{cases}
$$

Поскольку $\widehat{\delta}^{2}(y)=1, y \in Y$, то $\delta^{* 2}=E_{0}$. Продолжим функцию $\varphi(y)$, заданную на $E$, с сохранением ее свойств до функции $\widetilde{\varphi}(y)$ на $Y$ (см. [18, $\S 5.2])$. Пусть $\gamma$ - гауссовское распределение на $X$ с характеристической функцией $\widehat{\gamma}(y)=\left(x_{0}, y\right) \exp \{-\widetilde{\varphi}(y)\}$. Из того, что открытая подгруппа $E$ обладает свойством $E \cap Y^{(2)}=E^{(2)}$, следует, что $K=A(X, E)$ - компактная группа Корвина. Таким образом, из (3) и (13) вытекает, что $\widehat{\mu}(y)=\widehat{\gamma}(y) \widehat{m}_{K}(y) \widehat{\delta}(y)$, а значит, $\mu=\gamma * m_{K} * \delta$. Теорема доказана.

Отметим также, что если $X-$ произвольная локально компактная абелева группа и распределение $\mu \in M^{1}(X)$ имеет вид $\mu=\gamma * m_{K} * \delta$, где $\gamma \in \Gamma(X), K$ - компактная подгруппа Корвина, $\delta$ - заряд на $X_{(2)}$ такой, что $\delta^{* 2}=E_{0}$, то $\mu \in \Gamma_{\mathrm{B}}(X)$.

Из доказательства теоремы 1 непосредственно вытекает следующее утверждение.

Следствие 1. Пусть $X$ - локально компактная абелева группа. Предположим, ито связная компонента нуля $c_{X}$ группь $X$ содержит конечное число әлементов порядка 2. Пусть $\mu \in \Gamma_{\mathrm{B}}(X)$ и характеристическая функиия $\widehat{\mu}(y)$ не обращается в нуль. Тогда

$$
\mu=\gamma * \delta * E_{x},
$$

где $\gamma \in \Gamma^{\mathrm{s}}\left(c_{X}\right), \delta-$ заряд на $\left(c_{X}\right)_{(2)}$ такой, ито $\delta^{* 2}=E_{0}, x \in X$.

3 а м е ч а н и е 1 . Отметим роль элементов порядка 2 при изучении гауссовских распределений в смысле Бернштейна. Учитывая лемму 1 , будем предполагать, что группа $X$ имеет вид (5). Кроме того, предположим, что связная компонента нуля группы $X$ содержит конечное число элементов порядка 2. Как доказано в [9], следующие свойства группы $X$ эквивалентны:

(i) для любой компактной подгруппы Корвина $K$ группы $X$ факторгруппа $X / K$ не содержит подгруппы, топологически изоморфной двумерному тору $\mathbf{T}^{2}$;

(ii) связная компонента нуля $c_{X}$ группы $X$ содержит не более одного элемента порядка два, т.е. либо $\left(c_{X}\right)_{(2)}=\{0\}$, либо $\left(c_{X}\right)_{(2)} \cong \mathbf{Z}(2)$.

Поэтому, если связная компонента нуля группы $X$ содержит более одного элемента порядка 2 , то существует компактная подгруппа Корвина $K$ группы $X$ такая, что факторгруппа $X / K$ содержит подгруппу, топологически изоморфную двумерному тору $\mathbf{T}^{2}$. Нетрудно показать, что на группе $\mathbf{T}^{2}$ существуют гауссовское распределение $\gamma_{0}$ и 
заряд $\delta_{0}$, носитель которого совпадает с $\left(\mathbf{T}^{2}\right)_{(2)}$ и $\delta_{0}^{* 2}=E_{0}$ такие, что $\lambda=\gamma_{0} * \delta_{0} \in M^{1}\left(\mathbf{T}^{2}\right)$. Из (4) вытекает, что $\lambda \in \Gamma_{\mathrm{B}}\left(\mathbf{T}^{2}\right)$. Чтобы не вводить дополнительных обозначений, будем считать, что $\lambda \in M^{1}(X / K)$. Обозначим $H=A(Y, K)$. Поскольку $(X / K)^{*} \cong H$, то будем предполагать, что характеристическая функция $\widehat{\lambda}(y)$ определена на $H$. Положим

$$
f(y)= \begin{cases}\hat{\lambda}(y), & y \in H, \\ 0, & y \notin H .\end{cases}
$$

Легко видеть, что $f(y)$ - непрерывная положительно определенная функция на $Y$. По теореме Бохнера существует распределение $\mu \in$ $M^{1}(X)$ такое, что $\widehat{\mu}(y)=f(y)$. Из (4) следует, что $\mu \in \Gamma_{\mathrm{B}}(X)$. Из доказательства теоремы 1 вытекает, что $\mu=\gamma * m_{K} * \delta$, где $\gamma \in \Gamma(X)$, а $\delta$ - заряд на $X_{(2)}$ такой, что $\delta^{* 2}=E_{0}$. Таким образом, если связная компонента нуля группы $X$ содержит более одного элемента порядка 2 , то на группе $X$ существуют такие гауссовские распределения в смысле Бернштейна, при факторизации которых, помимо гауссовских распределений и распределений Хаара компактных подгрупп Корвина, присутствуют также заряды в носителем в $X_{(2)}$. Если же связная компонента нуля группы $X$ содержит не более одного элемента порядка 2, то, как доказано в [4] и [9], любое гауссовское распределение в смысле Бернштейна на группе $X$ представимо в виде свертки гауссовского распределения и распределения Хаара компактной подгруппы Корвина.

3. Носители гауссовских распределений в смысле Бернштейна. Целью этого пункта является доказательство следующего утверждения (ср. с леммой 1$)$.

Теорема 2. Пусть $X-$ локально компактная абелева группа. Пусть $\mu \in \Gamma_{\mathrm{B}}(X)$. Тогда носитель $\sigma(\mu)$ является классом смежности некоторой подгруппь в $X$, топологически изоморфной группе вида (5).

Доказательство теоремы 2 опирается на теорему 1 и следующую лемму.

Лемма 5. Пусть

$$
X=\mathbf{R}^{l} \times \mathbf{T}^{m} \times F,
$$

где $l \geqslant 0, m \geqslant 0$, а $F-$ конечная группа Корвина. Пусть распределение $\mu$ представимо в виде

$$
\mu=\gamma * m_{K} * \delta,
$$

где $\gamma \in \Gamma^{\mathrm{s}}\left(\mathbf{R}^{l} \times \mathbf{T}^{m}\right), K-$ компактная подгруппа Корвина в $X, \delta-$ заряд на $X_{(2)}$ такой, что $\delta^{* 2}=E_{0}$. Предположим, ито выполнено следующее условие:

(A) $X$ совпадает с минимальной замкнутой подгруппой, содержащей носитель $\sigma(\mu)$.

Тогда $\sigma(\mu)=X$. 
Д о к а з а т е л ь с т в о. Прежде чем переходить к доказательству леммы, сделаем следующее замечание. Как уже было отмечено, носитель $\sigma(\gamma)$ - некоторая связная подгруппа в $X$. Поскольку $\sigma\left(m_{K}\right)=K$, то $\sigma\left(\gamma * m_{K}\right)=\sigma(\gamma)+K$. Так как $F-$ конечная группа Корвина, то $F$ не содержит элементов порядка 2. Поэтому $X_{(2)} \subset \mathbf{T}^{m}$, откуда ввиду (A) следует, что $\sigma(\gamma)+K=X$, а значит, $\sigma\left(\gamma * m_{K}\right)=X$. Вообще говоря, если носитель некоторого распределения - вся группа, то при свертке этого распределения с зарядом в носителе свертки могут образоваться «дыры», т.е. носитель свертки не обязательно совпадает со всей группой. В данном же случае этого не происходит, что мы и докажем.

Поскольку $K-$ подгруппа в $\mathbf{T}^{m} \times F$, то очевидно, что группу $K$ можно представить в виде прямого произведения:

$$
K=A \times B,
$$

где $A \subset \mathbf{T}^{m}, A \cong \mathbf{T}^{a}, a \geqslant 0$, а $B-$ конечная группа. Отсюда следует, что

$$
m_{K}=m_{A} \otimes m_{B}=m_{A} * m_{B} .
$$

Представим группу $\mathbf{T}^{m}$ в виде $\mathbf{T}^{m}=\mathbf{T}^{m-a} \times \mathbf{T}^{a}$. Заметим теперь, что так как $A \cong \mathbf{T}^{a}$, то подгруппа $A$ выделяется в $\mathbf{T}^{m}$ как прямой сомножитель. Поэтому, не ограничивая общности, мы можем считать, что $A=\mathbf{T}^{a}$, а значит,

$$
m_{K}=m_{\mathbf{T}^{a}} * m_{B}
$$

Элементы группы $\mathbf{R}^{l} \times \mathbf{Z}^{m} \cong\left(\mathbf{R}^{l} \times \mathbf{T}^{m}\right)^{*}$ будем обозначать через $\left(s_{1}, \ldots, s_{l}, n_{1}, \ldots, n_{m}\right), s_{j} \in \mathbf{R}, n_{i} \in \mathbf{Z}$. Поскольку характеристическая функция $\widehat{m}_{\mathbf{T}^{a}}(y)$ имеет вид

$$
\widehat{m}_{\mathbf{T}^{a}}(y)= \begin{cases}1, & y \in A\left(Y, \mathbf{T}^{a}\right) \\ 0, & y \notin A\left(Y, \mathbf{T}^{a}\right)\end{cases}
$$

отсюда вытекает, что $\gamma * m_{\mathbf{T}^{a}}=\gamma_{1} * m_{\mathbf{T}^{a}}$, где $\gamma_{1} \in \Gamma^{\mathrm{s}}\left(\mathbf{R}^{l} \times \mathbf{T}^{m-a}\right)$, $\widehat{\gamma}_{1}\left(s_{1}, \ldots, s_{l}, n_{1}, \ldots, n_{m-a}\right)=\widehat{\gamma}\left(s_{1}, \ldots, s_{l}, n_{1}, \ldots, n_{m-a}, 0, \ldots, 0\right)$. Следовательно, $\gamma * m_{K}=\gamma_{1} * m_{K}$. Поэтому будем с самого начала предполагать, что в (14) $\gamma \in \Gamma^{\mathrm{s}}\left(\mathbf{R}^{l} \times \mathbf{T}^{m-a}\right)$.

По структурной теореме для конечных абелевых групп

$$
F \cong \mathbf{Z}\left(k_{1}\right) \times \cdots \times \mathbf{Z}\left(k_{n}\right),
$$

где $k_{j}$ - делитель $k_{j+1}, j=1,2, \ldots, n-1$. Чтобы не вводить новых обозначений, будем считать, что

$$
F=\mathbf{Z}\left(k_{1}\right) \times \cdots \times \mathbf{Z}\left(k_{n}\right) .
$$

Вложим группу $F$ естественным образом в $\mathbf{T}^{n}$, а группу $X$ будем рассматривать как подгруппу группы $\widetilde{X}=\mathbf{R}^{l} \times \mathbf{T}^{m} \times \mathbf{T}^{n}$. Обозначим через 
$p$ непрерывный гомоморфизм $p: \mathbf{R}^{l} \times \mathbf{R}^{m} \times \mathbf{R}^{n} \rightarrow \tilde{X}$, определяемый формулой

$$
p\left(t_{1}, \ldots, t_{l}, u_{1}, \ldots, u_{m}, v_{1}, \ldots, v_{n}\right)=\left(t_{1}, \ldots, t_{l}, e^{i u_{1}}, \ldots, e^{i u_{m}}, e^{i v_{1}}, \ldots, e^{i v_{n}}\right) .
$$

Пусть $\alpha \in \Gamma^{\mathrm{s}}\left(\mathbf{R}^{l} \times \mathbf{R}^{m-a}\right)$ - такое гауссовское распределение, что

$$
\gamma=p(\alpha) .
$$

Обозначим $L=\sigma(\alpha)$. Тогда $L-$ линейное подпространство в $\mathbf{R}^{l} \times$ $\mathbf{R}^{m-a} \subset \mathbf{R}^{l} \times \mathbf{R}^{m} \times \mathbf{R}^{n}$, а следовательно, подгруппа в $\mathbf{R}^{l} \times \mathbf{R}^{m} \times \mathbf{R}^{n}$.

Рассмотрим в $\mathbf{R}^{a} \subset \mathbf{R}^{m}$ куб

$$
Q=\left\{\left(u_{1}, \ldots, u_{a}\right), 0 \leqslant u_{j}<2 \pi, j=1,2, \ldots, a\right\} .
$$

Пусть $\beta$ обозначает меру Лебега на $Q$, нормированную таким образом, что $\beta(Q)=1$. Тогда, очевидно, $m_{\mathbf{T}^{a}}=p(\beta)$.

По структурной теореме для конечных абелевых групп группу $B$ можно представить в виде $B=B_{1} \times \cdots \times B_{b}$, где $B_{j} \cong \mathbf{Z}\left(l_{j}\right), j=1,2, \ldots, b$, и $l_{j}$ 一 делитель $l_{j+1}, j=1,2, \ldots, b-1$. Тогда существуют подгруппы $T_{j} \subset \widetilde{X}$ такие, что $B_{j} \subset T_{j}, T_{j} \cong \mathbf{T}, j=1,2, \ldots, b$. Мы имеем

$$
m_{B}=m_{B_{1}} \otimes \cdots \otimes m_{B_{b}}=m_{B_{1}} * \cdots * m_{B_{b}} .
$$

Обозначим через $C_{j}$ связную компоненту нуля группы $p^{-1}\left(T_{j}\right)$. Очевидно, что $C_{j} \cong \mathbf{R}$. Положим $E_{j}=C_{j} \cap p^{-1}\left(B_{j}\right)$. Тогда $E_{j} \cong \mathbf{Z}$. Пусть $e_{j}$ - образующая группы $E_{j}$. Рассмотрим множество $D_{j}=$ $\left\{0, e_{j}, 2 e_{j}, \ldots,\left(l_{j}-1\right) e_{j}\right\}$, и пусть $\omega_{j}$ обозначает равномерное распределение на $D_{j}$. Тогда $m_{B_{j}}=p\left(\omega_{j}\right)$. Положим $\omega=\omega_{1} * \cdots * \omega_{b}$. Тогда $m_{B}=p(\omega)$.

Обозначим через $P$ подгруппу в $\mathbf{R}^{l} \times \mathbf{R}^{m} \times \mathbf{R}^{n}$, порожденную подгруппами $L, \mathbf{R}^{a}$ и $E_{j}, j=1,2, \ldots, b$. Очевидно, что подгруппа $P$ топологически изоморфна группе вида $\mathbf{R}^{\widetilde{l}} \times \mathbf{Z}^{\tilde{m}}$. Положим $U=P \cap \operatorname{Ker} p$, $V=P / U$. Пусть $\tau-$ естественный гомоморфизм $\tau: P \rightarrow V$. Очевидно, что $V \cong \mathbf{R}^{p} \times \mathbf{T}^{d} \times G$, где $G-$ конечная группа. Легко видеть, что $G-$ группа Корвина. Чтобы не вводить новых обозначений, будем считать, что $V=\mathbf{R}^{p} \times \mathbf{T}^{d} \times G$. Очевидно, что группу $V$ можно заменить топологически изоморфной ей группой (в естественных обозначениях элементов группы $V$ это означает замену переменных) таким образом, что $V=\mathbf{R}^{p} \times \mathbf{T}^{q} \times \mathbf{T}^{a} \times G$, причем $\sigma(\tau(\alpha))=\mathbf{R}^{p} \times \mathbf{T}^{q}$ и $m_{\mathbf{T}^{a}}=\tau(\beta)$. Обозначим $\widetilde{\gamma}=\tau(\alpha)$. Очевидно, что гауссовское распределение $\widetilde{\gamma}$ и $m_{\mathbf{R}^{p} \times \mathbf{T}^{q}}$ взаимно абсолютно непрерывны. Пусть $\rho\left(t_{1}, \ldots, t_{p}, e^{i s_{1}}, \ldots, e^{i s_{q}}\right)$ - плотность $\widetilde{\gamma}$ относительно $m_{\mathbf{R}^{p} \times \mathbf{T}^{q}}$. Нетрудно проверить, что $\rho$ - целая функция относительно переменных $t_{i}, s_{j}$. Легко видеть, что свертка $\widetilde{\gamma} * m_{\mathbf{T}^{a}}$ имеет плотность

$$
\theta\left(t_{1}, \ldots, t_{p}, e^{i s_{1}}, \ldots, e^{i s_{q}}, e^{i u_{1}}, \ldots, e^{i u_{a}}\right)=\rho\left(t_{1}, \ldots, t_{p}, e^{i s_{1}}, \ldots, e^{i s_{q}}\right)
$$


относительно $m_{\mathbf{R}^{p} \times \mathbf{T}^{q} \times \mathbf{T}^{a}}$, которая является целой функцией от переменных $t_{i}, s_{j}, u_{k}$. Как видно из (16), $\theta$ зависит лишь от переменных $t_{i}, s_{j}$.

Обозначим $\widetilde{\omega}=\tau(\omega)$. Из конструкции $V$ следует, что $\widetilde{\omega}=m_{S}$, где $S$ - конечная подгруппа в $V$. Нетрудно также убедиться, что свертка $\widetilde{\gamma} * m_{\mathbf{T}^{a}} * m_{S}$ и $m_{V}$ взаимно абсолютно непрерывны.

Определим отображение $\widetilde{p}: V \rightarrow \widetilde{X}$ формулой $\widetilde{p}=p \tau^{-1}$. Очевидно, что $\widetilde{p}-$ непрерывный мономорфизм. Поскольку $\widetilde{p}(V) \subset X$, то $\widetilde{p}$ можно рассматривать как непрерывный мономорфизм из $V$ в $X$. Обозначим $M=\widetilde{p}(V) \subset X$.

Так как любая подгруппа в группе $X_{(2)}$ является в $X_{(2)}$ прямым сомножителем, то $X_{(2)}=M_{(2)} \times N$, где $N$ - некоторая подгруппа $X_{(2)}$. Поэтому каждый элемент группы $X_{(2)}$ может быть единственным образом записан в виде $x_{i j}=a_{i}+b_{j}, a_{i} \in M_{(2)}, b_{j} \in N$. Представим заряд $\delta$ в виде

$$
\delta=\sum_{i, j} c_{i j} E_{x_{i j}}=\sum_{i, j} c_{i j} E_{a_{i}} * E_{b_{j}}=\sum_{j} \pi_{j} * E_{b_{j}}
$$

где

$$
\pi_{j}=\sum_{i} c_{i j} E_{a_{i}}
$$

— некоторые заряды на $M_{(2)}$. Из (14) и (17) следует, что

$$
\mu=\sum_{j} \gamma * m_{K} * \pi_{j} * E_{b_{j}}
$$

Заметим, что заряды $\gamma * m_{K} * \pi_{j} * E_{b_{j}}$ сосредоточены на непересекающихся классах смежности $M+b_{j}$. Так как $\mu-$ мера, то отсюда следует, что все заряды $\gamma * m_{K} * \pi_{j}$ - также меры.

С другой стороны, поскольку $\delta^{* 2}=E_{0}$, то $\mu^{* 2}=\gamma^{* 2} * m_{K}$. Следовательно, распределение $\mu^{* 2}$ сосредоточено на $M$. Отсюда вытекает, что в (18) все свертки $\gamma * m_{K} * \pi_{j}$, кроме одной, должны быть равны нулю. Итак, мы имеем

$$
\mu=\gamma * m_{K} * \delta=\gamma * m_{K} *\left(\sum_{i} c_{i j_{0}} E_{a_{i}}\right) * E_{b_{j_{0}}} .
$$

Предположим вначале, что в (19) $b_{j_{0}}=0$, т.е.

$$
\mu=\gamma * m_{K} * \pi_{j_{0}} .
$$

Рассмотрим на $V$ заряд $\zeta=\widetilde{p}^{-1}\left(\pi_{j_{0}}\right)$. Поскольку $\gamma=\widetilde{p}(\widetilde{\gamma}), m_{\mathbf{T}^{a}}=$ $\widetilde{p}\left(m_{\mathbf{T}^{a}}\right), m_{B}=\widetilde{p}\left(m_{S}\right), \pi_{j_{0}}=\widetilde{p}(\zeta)$, то из $(15)$ и (20) мы получаем

$$
\mu=\gamma * m_{K} * \pi_{j_{0}}=\gamma * m_{\mathbf{T}^{a}} * m_{B} * \pi_{j_{0}}=\widetilde{p}\left(\widetilde{\gamma} * m_{\mathbf{T}^{a}} * m_{S} * \zeta\right) .
$$


Пусть $c$ обозначат число элементов группы $S$. Каждый элемент группы $S$ можно представить в виде $s_{i j}=c_{i}+d_{j}$, где $c_{i} \in \mathbf{T}^{q} \times \mathbf{T}^{a}, d_{j} \in G$. Имеем

$$
m_{S}=\frac{1}{c} \sum_{i, j} E_{s_{i j}}=\frac{1}{c} \sum_{i, j} E_{c_{i}} * E_{d_{j}}=\sum_{j} \kappa * E_{d_{j}},
$$

где $\kappa=(1 / c) \sum_{i} E_{c_{i}}$. Следовательно,

$$
\widetilde{\gamma} * m_{\mathbf{T}^{a}} * m_{S} * \zeta=\sum_{j} \widetilde{\gamma} * m_{\mathbf{T}^{a}} * \kappa * \zeta * E_{d_{j}} .
$$

Поскольку $\widetilde{p}$ - мономорфизм, то из (21) вытекает, что заряд $\widetilde{\gamma} *$ $m_{\mathbf{T}^{a}} * m_{S} * \zeta$ на самом деле является мерой. Заметим, что заряд $\widetilde{\gamma} *$ $m_{\mathbf{T}^{a}} * \kappa * \zeta$ сосредоточен на подгруппе $\mathbf{R}^{p} \times \mathbf{T}^{q} \times \mathbf{T}^{a}$, а следовательно, заряды $\widetilde{\gamma} * m_{\mathbf{T}^{a}} * \kappa * \zeta * E_{d_{j}}$ сосредоточены на непересекающихся классах смежности $\mathbf{R}^{p} \times \mathbf{T}^{q} \times \mathbf{T}^{a}+d_{j}$. Отсюда следует, что заряд $\widetilde{\gamma} * m_{\mathbf{T}^{a}} *$ $\kappa * \zeta$ также является мерой. Из (16) следует, что мера $\widetilde{\gamma} * m_{\mathbf{T}^{a}} * \kappa * \zeta$ имеет плотность относительно $m_{V}$, которая является целой функцией от переменных $t_{i}, s_{j}, u_{k}$. Обозначим эту плотность через $\lambda$. Поскольку $\lambda$ - целая функция, то

$$
\begin{aligned}
& m_{\mathbf{R}^{p+q+a}}\left\{\left(t_{1}, \ldots, t_{p}, s_{1}, \ldots, s_{q}, u_{1}, \ldots, u_{a}\right) \in \mathbf{R}^{p+q+a}:\right. \\
&\left.\lambda\left(t_{1}, \ldots, t_{p}, e^{i s_{1}}, \ldots, e^{i s_{q}}, e^{i u_{1}}, \ldots, e^{i u_{a}}\right)=0\right\}=0
\end{aligned}
$$

(см., например, $[19$, гл. $2, \S 2])$. Следовательно, $\widetilde{\gamma} * m_{\mathbf{T}^{a}} * m_{S} * \zeta$ и $m_{V}$ взаимно абсолютно непрерывны. Учитывая (21), отсюда вытекает, что $\sigma(\mu)=\overline{\widetilde{p}(V)}$. Принимая во внимание условие $(\mathrm{A})$, заключаем, что $\sigma(\mu)=$ $X$. Лемма, в случае, когда в (19) $b_{j_{0}}=0$, доказана.

Если в $(19) b_{j_{0}} \neq 0$, то рассуждение, проведенное выше для случая $b_{j_{0}}=0$, нужно применить к распределению $\nu=\mu * E_{b_{j_{0}}}$. Мы получаем, что $\sigma(\nu)=\overline{\widetilde{p}(V)}$. Отсюда вытекает, что

$$
\sigma(\mu)=\overline{\widetilde{p}(V)}+b_{j_{0}} .
$$

Если $\overline{\widetilde{p}(V)} \neq X$, то из вида группы $X$ и (23) следует, что минимальная замкнутая подгруппа в $X$, содержащая $\sigma(\mu)$, является собственной подгруппой в $X$, что противоречит условию (А). Таким образом, $\overline{\widetilde{p}(V)}=X$. Следовательно, $\sigma(\mu)=X$, а значит, лемма полностью доказана.

Доказ а тельств о т е о ремы 2. Пусть $\mu \in \Gamma_{\mathrm{B}}(X)$. Обозначим

$$
B=\{y \in Y:|\widehat{\mu}(y)|=1\} .
$$

Заметим, прежде всего, что, изучая носитель $\mu$, можно предполагать, что $B=\{0\}$. Действительно, $B$ является подгруппой. Поскольку характеристическая функция $\widehat{\mu}(y)$ на $B$ по модулю равна 1 , то существует 
такой элемент $x \in X$, что $\widehat{\mu}(y)=(x, y), y \in B$. Положим $\nu=\mu * E_{-x}$. Очевидно, $\nu \in \Gamma_{B}(X)$ и $\nu$ обладает тем свойством, что

$$
\{y \in Y: \widehat{\nu}(y)=1\}=B .
$$

Обозначим $G=A(X, B), H=G^{*}$. Тогда $\sigma(\nu) \subset G$, причем

$$
\{h \in H:|\widehat{\nu}(h)|=1\}=\{0\},
$$

поскольку

$$
\{h \in H: \widehat{\nu}(h)=1\}=\{h \in H:|\widehat{\nu}(h)|=1\} .
$$

Сделанное выше замечание и лемма 1 показывают, что мы можем предполагать, что группа $X$ имеет вид (5), а распределение $\mu$ удовлетворяет условию

$$
\{y \in Y:|\widehat{\mu}(y)|=1\}=\{0\} .
$$

Теорема будет доказана, если мы проверим, что $\mu(U)>0$ для любого открытого подмножества $U \subset X$. Допустим противное. Тогда $\mu(U)=0$ для некоторого открытого множества $U \subset X$. Выберем такое открытое подмножество $U_{0}$ в $U$ и такую окрестность нуля $V$ в $X$, что $U_{0}+V \subset U$. Пересечение $K \cap V$ является окрестностью нуля группы $K$. Поэтому существует такая замкнутая подгруппа $S \subset K \cap V$, что $K / S \cong \mathbf{T}^{m} \times F$, где $m \geqslant 0$, а $F-$ конечная группа. Очевидно, что $F$ - группа Корвина. Имеем в результате $X / S \cong M$, где

$$
M=\mathbf{R}^{l} \times \mathbf{T}^{m} \times F .
$$

Обозначим этот топологический изоморфизм через $\tau$ и положим $p=\tau \pi$, где $\pi$ - естественный гомоморфизм $\pi: X \rightarrow X / S$. Таким образом,

$$
p: X \rightarrow M \text {. }
$$

Обозначим $N=M^{*}$. Очевидно, что $p(\mu) \in \Gamma_{\mathrm{B}}(M)$, причем из (24) следует, что

$$
\{y \in N:|\widehat{p(\mu)}(y)|=1\}=\{0\} .
$$

Так как связная компонента нуля группы $M$, очевидно, содержит конечное число элементов порядка 2 , то по теореме 1 распределение $p(\mu)$ представимо в виде

$$
p(\mu)=\gamma * m_{K} * \delta * E_{x_{0}},
$$

где $\gamma \in \Gamma^{\mathrm{s}}\left(\mathbf{R}^{l} \times \mathbf{T}^{m}\right), K-$ компактная подгруппа Корвина в $M, \delta-$ заряд на $M_{(2)}$ такой, что $\delta^{* 2}=E_{0}, x_{0} \in M$. Положим $\alpha=p(\mu) * E_{-x_{0}}$. Тогда $\alpha \in \Gamma_{\mathrm{B}}(M)$. Из (25) следует, что

$$
\{y \in N:|\widehat{\alpha}(y)|=1\}=\{0\} .
$$


Отсюда, в частности, вытекает, что минимальная замкнутая подгруппа в $M$, содержащая носитель $\sigma(\alpha)$, совпадает с $M$. Применяя к распределению $\alpha$ лемму 5 , получаем $\sigma(\alpha)=M$. Следовательно,

$$
\sigma(p(\mu))=M
$$

С другой стороны, из нашей конструкции следует, что

$$
p(\mu)\left(U_{0}\right)=\mu\left\{p^{-1}\left(p\left(U_{0}\right)\right)\right\}=\mu\left\{U_{0}+S\right\} \leqslant \mu\left\{U_{0}+V\right\} \leqslant \mu\{U\}=0,
$$

что противоречит (26), ибо $p$ - открытое отображение, а значит, множество $p\left(U_{0}\right)$ открыто. Итак, $\mu(U)>0$. Теорема доказана.

Отметим также, что если $X$ - произвольная локально компактная абелева группа, топологически изоморфная группе вида (5), то существует распределение $\mu \in \Gamma_{\mathrm{B}}(X)$ такое, что $\sigma(\mu)=X$.

3 а м е ч а н и е 2. Учитывая, что $\Gamma(X) \subset \Gamma_{\mathrm{B}}(X)$, сравним теорему 2 с соответствующим результатом о носителе гауссовского распределения. Напомним, что носитель симметричного гауссовского распределения - некоторая связная подгруппа в $X$. По структурной теореме для связных локально компактных абелевых групп каждая такая группа топологически изоморфна группе вида $\mathbf{R}^{l} \times K$, где $l \geqslant 0$, а $K-$ связная компактная группа. В свою очередь, компактная группа $K$ связна тогда и только тогда, когда $K^{(n)}=K$ для всех натуральных $n$. Поэтому связная компактная группа является группой Корвина, а множество связных групп $X$ является подмножеством в множестве групп вида (5).

4. Закон нуля или единицы для гауссовских распределений в смысле Бернштейна. Распределение $\mu_{1}$ на группе $X$ называется делителем распределения $\mu$, если $\mu=\mu_{1} * \mu_{2}$, где $\mu_{2} \in M^{1}(X)$. Распределение $\mu$ на группе $X$ называется идемпотентным, если $\mu^{* 2}=\mu * E_{x}$ при некотором $x \in X$. Хорошо известно, что множество идемпотентных распределений на группе $X$ совпадает с множеством сдвигов распределений Хаара компактных подгрупп группы $X$ (см. [16]).

Основным результатом этого пункта является доказательство следующего утверждения.

Теорема 3. Пусть $X-$ локально компактная абелева группа, компонента нуля которой имеет конечную размерность. Пусть $\mu \in$ $\Gamma_{\mathrm{B}}(X)$ и н не имеет идемпотентных делителей. Тогда для любой борелевской подгруппь $G \subset X$ либо $\mu(G)=0$, либо $\mu(G)=1$.

Для доказательства теоремы 3 нам понадобятся следующие леммы.

Лемма 6. Пусть $X-$ локально компактная абелева группа и $\mu \in$ $\Gamma_{\mathrm{B}}(X)$. Распределение $\mu$ не имеет идемпотентных делителей тогда и только тогда, когда $\widehat{\mu}(y) \neq 0$ для всех $y \in Y$. 
Д о к а з а т е л ь с т в о. Обозначим $E=\{y \in Y: \widehat{\mu}(y) \neq 0\}$. Из (4) следует, что $E$ - подгруппа в $Y$, очевидно, открытая. Тогда ее аннулятор $K=A(X, E)$ - компактная подгруппа в $X$. Из (3) получаем, что $\widehat{\mu}(y)=\widehat{\mu}(y) \widehat{m}_{K}(y)$, а следовательно, $\mu=\mu * m_{K}$. Отсюда непосредственно вытекает утверждение леммы.

Лемма 7 (см. [20]). Пусть $X-$ связная локально компактная абелева группа конечной размерности $l$ u $\gamma \in \Gamma^{\mathrm{s}}(X)$. Тогда существуют непрерьвныи гомоморфизм $q: \mathbf{R}^{l} \rightarrow X$ и гауссовское распределение $\alpha \in \Gamma^{\mathrm{s}}\left(\mathbf{R}^{l}\right)$ такое, ито $\gamma=q(\alpha)$.

Лемма 8. Пусть $X=\mathbf{R}^{n} \times \mathbf{T}^{m} \times D$, где $D-$ дискретная счетнал абелева группа, $\gamma \in \Gamma(X)$ и $\gamma$ абсолютно непрерывно относительно $m_{X}$. Пусть $\delta$ - заряд на $\mathbf{R}^{n} \times \mathbf{T}^{m}$, сосредоточенный в конечном числе точек, $u \mu=\gamma * \delta \in M^{1}(X)$. Тогда $\mu$ и $\gamma$ взаимно абсолютно непрерывны.

Д о к а з а т е л ь с т в о. Мы ограничимся доказательством леммы для случая группы $X=\mathbf{T}^{m}$. Общий случай рассматривается аналогично. Очевидно, что, не ограничивая общности, гауссовское распределение $\gamma$ можно считать симметричным. А тогда, как следует из определения гауссовского распределения, характеристическая функция $\widehat{\gamma}(k)$ может быть записана в виде

$$
\widehat{\gamma}(k)=\exp \left\{-\frac{1}{2}\langle A k, k\rangle\right\}, \quad k \in \mathbf{Z}^{m},
$$

где $A$ - симметричная неотрицательно определенная матрица, а $\langle\cdot, \cdot\rangle-$ скалярное произведение в $\mathbf{R}^{m}$. Так как распределение $\gamma$ абсолютно непрерывно относительно $m_{X}$, то $\operatorname{det} A \neq 0$. Очевидно, что $\gamma$ и $m_{X}$ взаимно абсолютно непрерывны и $\mu$ абсолютно непрерывно относительно $\gamma$. Докажем, что $\gamma$ абсолютно непрерывно относительно $\mu$. Легко видеть, что плотность $\rho\left(e^{i t}\right)$ распределения $\gamma$ относительно распределения Хаара $m_{X}$ может быть записана в виде

$$
\rho\left(e^{i t}\right)=\sqrt{\frac{(2 \pi)^{m}}{\operatorname{det} A}} \sum_{k \in \mathbf{Z}^{m}} \exp \left\{-\frac{1}{2}\left\langle A^{-1}(t+2 \pi k), t+2 \pi k\right\rangle\right\},
$$

где $t=\left(t_{1}, \ldots, t_{m}\right) \in \mathbf{R}^{m}, e^{i t}=\left(e^{i t_{1}}, \ldots, e^{i t_{m}}\right) \in \mathbf{T}^{m}$. В частности, при $m=1$, если мы записываем характеристическую функцию $\widehat{\gamma}(k)$ в виде

$$
\widehat{\gamma}(k)=\exp \left\{-\frac{\sigma^{2} k^{2}}{2}\right\}, \quad k \in \mathbf{Z}
$$

то мы имеем

$$
\rho\left(e^{i t}\right)=\frac{\sqrt{2 \pi}}{\sigma} \sum_{n=-\infty}^{\infty} \exp \left\{-\frac{1}{2 \sigma^{2}}(t+2 \pi n)^{2}\right\}, \quad t \in \mathbf{R} .
$$


Функция $\rho\left(e^{i t}\right)$ продолжается формулой $(27)$ в $\mathbf{C}^{m}$ как целая функция по $t$. Действительно, если $f(t)-$ произвольная функция от $t=$ $\left(t_{1}, \ldots, t_{m}\right) \in \mathbf{R}^{m}$, то $f(t)$ продолжается в $\mathbf{C}^{m}$ как целая функция по $t$ тогда и только тогда, когда продолжается в $\mathbf{C}^{m}$ функция $g(s)=f(t)$, $s=T t$, где $T$ - некоторая обратимая матрица. Поэтому в (27) матрицу $A$ можно считать диагональной, а тогда доказательство возможности для функции $\rho\left(e^{i t}\right)$ быть продолженной сводится к одномерному случаю. Но если $m=1$, то из (28), очевидно, следует, что функция $\rho\left(e^{i t}\right)$ продолжается формулой $(28)$ в $\mathbf{C}$ как целая функция по $t$.

Пусть $\delta=\sum c_{j} E_{x_{j}}$, где $c_{j} \in \mathbf{R}, x_{j}=e^{i a_{j}} \in \mathbf{T}^{m}$. Тогда распределение $\mu$ имеет плотность

$$
r\left(e^{i t}\right)=\sum c_{j} \rho\left(e^{i\left(t-a_{j}\right)}\right)
$$

относительно $m_{X}$, которая также является целой функцией по $t$. Отсюда следует, что

$$
m_{\mathbf{R}^{m}}\left\{t \in \mathbf{R}^{m}: r\left(e^{i t}\right)=0\right\}=0
$$

(см., например, $[19$, гл. $2, \S 2])$. Следовательно, мера Хаара $m_{X}$ абсолютно непрерывна относительно $\mu$, а значит, и $\gamma$ абсолютно непрерывно относительно $\mu$. Лемма доказана.

Д ок аз а т ель с т в о т е о ре мы 3 . Заметим, что если $c_{X}$ имеет конечную размерность, то группа $c_{X}$ содержит конечное число элементов порядка 2. Действительно, по структурной теореме для связных локально компактных абелевых групп мы имеем $c_{X} \cong \mathbf{R}^{p} \times K$, где $p \geqslant 0$, а $K$ - связная компактная абелева группа. Поэтому будем считать, что $c_{X}-$ компактная группа. Обозначим $H=\left(c_{X}\right)^{*}$. Тогда $H-$ дискретная группа без кручения и размерность $c_{X}$ совпадает с рангом $r(H)$. Следовательно, $r(H)<\infty$. Заметим, что $A\left(H,\left(c_{X}\right)_{(2)}\right)=H^{(2)}$. Отсюда следует $\left(\left(c_{X}\right)_{(2)}\right)^{*} \cong H / H^{(2)}$. Все ненулевые элементы факторгруппы $H / H^{(2)}$ имеют порядок 2. Поэтому мы можем рассматривать факторгруппу $H / H^{(2)}$ как линейное пространство над полем $\mathbf{Z}(2)$ и его размерность не превосходит $r(H)$. Отсюда вытекает, что группа $\left(\left(c_{X}\right)_{(2)}\right)$ * конечна, а значит, и группа $\left(c_{X}\right)_{(2)}$ конечна.

По следствию 1 распределение $\mu$ может быть представлено в виде

$$
\mu=\gamma * \delta * E_{x}
$$

где $\gamma \in \Gamma^{\mathrm{s}}\left(c_{X}\right), \delta$ - заряд на $\left(c_{X}\right)_{(2)}$ такой, что $\delta^{* 2}=E_{0}$ и $x \in X$. По лемме $7 \gamma=q(\alpha)$, где $q: \mathbf{R}^{l} \rightarrow c_{X} \subset X-$ непрерывный гомоморфизм, а $\alpha \in \Gamma^{\mathrm{s}}\left(\mathbf{R}^{l}\right)$. Очевидно, можно считать, что $\sigma(\alpha)=\mathbf{R}^{l}$, а тогда гауссовское распределение $\alpha$ и мера Хаара $m_{\mathbf{R}^{l}}$ взаимно абсолютно непрерывны. Обозначим $N=\operatorname{Ker} q$. Тогда $N-$ замкнутая подгруппа в $\mathbf{R}^{l}$, а следовательно, $N \cong \mathbf{R}^{a} \times \mathbf{Z}^{m}$. Пусть $\pi: \mathbf{R}^{l} \rightarrow \mathbf{R}^{l} / N-$ естественный гомоморфизм. Мы имеем, $\mathbf{R}^{l} / N \cong \mathbf{R}^{n} \times \mathbf{T}^{m}$. Чтобы не вводить 
новых обозначений, будем считать. что $\mathbf{R}^{l} / N=\mathbf{R}^{n} \times \mathbf{T}^{m}$. Гомоморфизм $q$ индуцирует непрерывный мономорфизм

$$
p: \mathbf{R}^{n} \times \mathbf{T}^{m} \rightarrow X
$$

по формуле $p=q \pi^{-1}$. Тогда $\gamma=p(\beta)$, где $\beta=\pi(\alpha) \in \Gamma^{\mathrm{s}}\left(\mathbf{R}^{n} \times \mathbf{T}^{m}\right)$. Отметим, что гауссовское распределение $\beta$ и мера Хаара $m_{\mathbf{R}^{n} \times \mathbf{T}^{m}}$ также взаимно абсолютно непрерывны. Обозначим $F=p\left(\mathbf{R}^{n} \times \mathbf{T}^{m}\right), A=$ $\left(c_{X}\right)_{(2)} \cap F$. Заметим, что $F-$ некоторая борелевская подгруппа в $X$. Мы имеем $\left(c_{X}\right)_{(2)}=A \times B$, где $B-$ некоторая подгруппа $\left(c_{X}\right)_{(2)}$. Тогда каждый элемент подгруппы $\left(c_{X}\right)_{(2)}$ может быть единственным образом записан в виде $x_{i j}=a_{i}+b_{j}, a_{i} \in A, b_{j} \in B$.

Пусть заряд $\delta$ имеет вид

$$
\delta=\sum_{i, j} c_{i j} E_{x_{i j}}=\sum_{i, j} c_{i j} E_{a_{i}} * E_{b_{j}}=\sum_{j}\left(\sum_{i} c_{i j} E_{a_{i}}\right) * E_{b_{j}} .
$$

Тогда

$$
\mu=\left(\sum_{j} \gamma * \omega_{j} * E_{b_{j}}\right) * E_{x}
$$

где $\omega_{j}=\sum_{i} c_{i j} E_{a_{i}}$ - некоторые заряды на $F$. Отметим, что распределение $\gamma$ также сосредоточено на $F$. Поскольку $\mu-$ мера, то все свертки $\gamma * \omega_{j}$ также меры, так как заряды $\gamma * \omega_{j} * E_{b_{j}}$ сосредоточены на непересекающихся классах смежности $F+b_{j}$.

Воспользуемся теперь тем, что $\delta^{* 2}=E_{0}$. Из (29) получаем, что $\mu^{* 2}=\gamma^{* 2} * E_{2 x}$. Следовательно, распределение $\mu^{* 2}$ сосредоточено на множестве $F+2 x$.

Предположим, что хотя бы две меры $\gamma * \omega_{j_{1}}$ и $\gamma * \omega_{j_{2}}$ ненулевые. Из (30) следует тогда, что

$$
\mu^{* 2}\left(\left\{F+2 x+b_{j_{1}}+b_{j_{2}}\right\}\right)>0 .
$$

Поскольку множества $F$ и $F+b_{j_{1}}+b_{j_{2}}$ не пересекаются, это неравенство противоречит тому, что распределение $\mu^{* 2}$ сосредоточено на множестве $F+2 x$. Таким образом, все свертки $\gamma * \omega_{j}$, кроме одной, равны нулю. Поскольку $\widehat{\gamma}(y) \neq 0$ при всех $y \in Y$, получаем, что $\omega_{j}=0$ при всех $j$, кроме некоторого $j=j_{0}$. Таким образом,

$$
\delta=\left(\sum_{i} c_{i j_{0}} E_{a_{i}}\right) * E_{b_{j_{0}}} .
$$

Из (29) и (31) вытекает, что

$$
\mu=\gamma *\left(\sum_{i} c_{i j_{0}} E_{a_{i}}\right) * E_{x+b_{j_{0}}}
$$


Обозначим $\widetilde{x}=x+b_{j_{0}}$. Возможны два случая: $\widetilde{x} \in F$ и $\widetilde{x} \notin F$.

Случай 1: $\widetilde{x} \in F$. Из (31) вытекает, что заряд $\delta * E_{x}$ сосредоточен на $F$, а следовательно, распределение $\mu=\gamma * \delta * E_{x}$ также сосредоточено на $F$. Обозначим $\lambda=p^{-1}\left(\delta * E_{x}\right)$. Тогда $\lambda-$ заряд на $\mathbf{R}^{n} \times \mathbf{T}^{m}$. Мы имеем $\mu=p(\nu)$, где $\nu=\beta * \lambda$. Пусть $G-$ борелевская подгруппа $X$ и $\mu(G)>0$. Тогда $\nu\left(p^{-1}(G)\right)>0$. Из леммы 8 вытекает, что распределения $\nu$ и $\beta$ взаимно абсолютно непрерывны. Поэтому $\beta\left(p^{-1}(G)\right)>0$. Так как гауссовское распределение $\beta$ абсолютно непрерывно относительно $m_{\mathbf{R}^{n} \times \mathbf{T}^{m}}$, то $m_{\mathbf{R}^{n} \times \mathbf{T}^{m}}\left(p^{-1}(G)\right)>0$. Поскольку $p^{-1}(G)$ - борелевская подгруппа в $\mathbf{R}^{n} \times \mathbf{T}^{m}$ и $m_{\mathbf{R}^{n} \times \mathbf{T}^{m}}\left(p^{-1}(G)\right)>0$, то подгруппа $p^{-1}(G)$ содержит окрестность нуля группы $\mathbf{R}^{n} \times \mathbf{T}^{m}$. Учитывая, что группа $\mathbf{R}^{n} \times \mathbf{T}^{m}$ связна, получаем $p^{-1}(G)=\mathbf{R}^{n} \times \mathbf{T}^{m}$. Следовательно, $G=F$, и, значит, $\mu(G)=\mu(F)=1$. В случае 1 теорема доказана.

Случай 2: $\widetilde{x} \notin F$. Обозначим $\widetilde{\gamma}=\gamma * E_{\widetilde{x}}$ и перепишем (32) в виде

$$
\mu=\widetilde{\gamma} * \omega_{j_{0}} .
$$

Пусть $q-$ непрерывный гомоморфизм $q: \mathbf{R}^{l} \rightarrow c_{X}$, существующий по лемме 7 , такой, что $\gamma=q(\alpha)$, где $\alpha \in \Gamma^{\mathrm{s}}\left(\mathbf{R}^{l}\right)$ и $\sigma(\alpha)=\mathbf{R}^{l}$. Очевидно, что $F=q\left(\mathbf{R}^{l}\right)$. Возможны два случая: $n \widetilde{x} \in F$ для некоторого натурального $n \geqslant 2$ и $n \widetilde{x} \notin F$ для всех натуральных $n$.

a. Предположим, что $n_{0} \widetilde{x}=q\left(t_{0}\right) \in F$ при некотором натуральном $n_{0} \geqslant 2$. Будем считать, что это $n_{0}$ наименьшее. Положим $x_{0}=\widetilde{x}-q\left(\frac{t_{0}}{n_{0}}\right)$. Тогда $n_{0} x_{0}=0$. Продолжим $q$ до гомоморфизма

$$
\widetilde{q}: \mathbf{R}^{l} \times \mathbf{Z}\left(n_{0}\right) \rightarrow X
$$

полагая

$$
\widetilde{q}(t, k)=q(t)+k x_{0}, \quad t \in \mathbf{R}^{l}, \quad k=0,1, \ldots, n_{0}-1 .
$$

Заметим, что $N=\operatorname{Ker} q=\operatorname{Ker} \widetilde{q}$. Легко видеть, что $\left(\mathbf{R}^{l} \times\right.$ $\left.\mathbf{Z}\left(n_{0}\right)\right) / N \cong \mathbf{R}^{n} \times \mathbf{T}^{m} \times \mathbf{Z}\left(n_{0}\right)$. Пусть $\pi: \mathbf{R}^{l} \times \mathbf{Z}\left(n_{0}\right) \rightarrow\left(\mathbf{R}^{l} \times \mathbf{Z}\left(n_{0}\right)\right) / N-$ естественный гомоморфизм. Обозначим $L=\mathbf{R}^{n} \times \mathbf{T}^{m} \times \mathbf{Z}\left(n_{0}\right)$. Чтобы не вводить новых обозначений, будем считать, что $\left(\mathbf{R}^{l} \times \mathbf{Z}\left(n_{0}\right)\right) / N=L$. Гомоморфизм $\widetilde{q}$ индуцирует непрерывный мономорфизм

$$
p: L \rightarrow X
$$

по формуле $p=\widetilde{q} \pi^{-1}$. Тогда $\widetilde{\gamma}=p(\widetilde{\beta})$, где $\widetilde{\beta}=\pi\left(\alpha * E_{\left(t_{0} / n_{0}, 1\right)}\right) \in \Gamma(L)$. Отметим, что гауссовское распределение $\widetilde{\beta}$ абсолютно непрерывно относительно $m_{L}$. Обозначим $\lambda=p^{-1}\left(\omega_{j_{0}}\right)$. Тогда $\lambda-$ заряд на $\mathbf{R}^{n} \times \mathbf{T}^{m}$. Из (33) следует, что $\mu=p(\nu)$, где $\nu=\widetilde{\beta} * \lambda$.

Пусть $G$ - борелевская подгруппа $X$ и $\mu(G)>0$. Тогда $\nu\left(p^{-1}(G)\right)>$ 0. Из леммы 8 вытекает, что распределения $\nu$ и $\widetilde{\beta}$ взаимно абсолютно 
непрерывны. Поэтому $\widetilde{\beta}\left(p^{-1}(G)\right)>0$. Следовательно, $m_{L}\left(p^{-1}(G)\right)>0$. Поскольку $p^{-1}(G)$ - борелевская подгруппа в $L$ и $m_{L}\left(p^{-1}(G)\right)>0$, то подгруппа $p^{-1}(G)$ содержит окрестность нуля группы $L$. Учитывая, что группа $\mathbf{R}^{n} \times \mathbf{T}^{m}$ связна, получаем $p^{-1}(G) \supset \mathbf{R}^{n} \times \mathbf{T}^{m}$. Следовательно, $G \supset F$. Из (32) следует, что распределение $\mu$ сосредоточено на классе смежности $\widetilde{x}+F$. Поэтому если $\mu(G)>0$, то $\{\widetilde{x}+F\} \cap G \neq \emptyset$. Так как $G \supset F$, то отсюда вытекает, что $G \supset\{\widetilde{x}+F\}$. Следовательно, $\mu(G) \geqslant \mu(\{\widetilde{x}+F\})=1$. В случае 2 а теорема также доказана.

b. Если $n \tilde{x} \notin F$ при всех натуральных $n$, то рассуждение аналогично проведенному выше. Единственное отличие состоит в том, что $q$ продолжаем до гомоморфизма

$$
\widetilde{q}: \mathbf{R}^{l} \times \mathbf{Z} \rightarrow X
$$

полагая

$$
\widetilde{q}(t, k)=q(t)+k \widetilde{x}, \quad t \in \mathbf{R}^{l}, \quad k \in \mathbf{Z} .
$$

Теорема полностью доказана.

Автор благодарит рецензента за полезные замечания.

Работа выполнена в рамках проекта «Украинский филиал французскороссийской лаборатории им. Ж.-В. Понселе «Вероятностные задачи на группах и в спектральной теории»».

\section{СПИСОК ЛИТЕРАТУРЫ}

1. Рухин А. Л. Об одной теореме С. Н. Бернштейна. - Матем. заметки, 1969, т. 6, № 3, с. 301-307.

2. Рухин А.Л. Некоторые статистические и вероятностные задачи на группах. Тр. МИАН, 1970, т. 111, с. $52-109$.

3. Heyer H., Rall Ch. Gauss'she Wahrscheinlichkeitsmasse auf Corwinschen Gruppen. Math. Z., 1972, v. 128, № 4, p. 343-361.

4. Фельдман Г. М. Гауссовские распределения в смысле Бернштейна на группах. Теория вероятн. и ее примен., 1986 , т. 31 в. в, с. 47-58.

5. Baryshnikov Y., Eisenberg B., Stadje $W$. Independent variables with independent sum and difference: $S^{1}$-case. - J. Multivariate Anal., 1993, v. 45, № 2, p. 161-170.

6. Neuenschwander D. Gauss measures in the sense of Bernstein on the Heisenberg group. - Probab. Math. Statist., 1993, v. 14, № 2, p. 253-256.

7. Franz U., Neuenschwander D., Schott R. Gauss laws in the sense of Bernstein and uniqueness of embedding into convolution semigroups on quantum groups and braided groups. - Probab. Theory Related Fields, 1997, v. 109, № 1, p. 101-127.

8. Neuenschwander D., Schott R. The Bernstein and Skitovič-Darmois characterization theorems for Gaussian distributions on groups, symmetric spaces, and quantum groups. - Exposition. Math., 1997, v. 15, № 4, p. 289-314.

9. Feldman G.M. On groups for which the Bernstein characterization of a Gaussian distribution is valid. - Доповіді Нацональної академї наук України. Математика. Природознавство. Технічні науки, 2001, № 8, с. 29-32.

10. Myronyuk $M$. An analogue of the Bernstein theorem for the cylinder. - Aequationes Math., 2006, v. 71, № 1-2, p. 54-69. 
11. Feldman G. M. Functional Equations and Characterization Problems on Locally Compact Abelian Groups. Zürich: Eur. Math. Soc., 2008, 268 p. (EMS Tracts Math., v. 5.)

12. Byczkowski T. Gaussian measures on metric abelian groups. - Lecture Notes in Math., 1979, v. 706, p. 41-53.

13. Byczkowski T. Zero-one laws for Gaussian measures on metric abelian groups. Studia Math., 1980/81, v. 69, № 2, p. 159-189.

14. Byczkowska H., Byczkowski T. A generalization of the zero-one law for Gaussian measures on metric abelian groups. - Bull. Acad. Polon. Sci., 1981, v. 29, № 3-4, p. $187-191$

15. Хьюитm Э., Росс К. Абстрактный гармонический анализ, т. 1. М.: Наука, 1975, $656 \mathrm{c}$.

16. Parthasarathy K.R., Ranga Rao R., Varadhan S.R.S. Probability distributions on locally compact Abelian groups. - Illinois J. Math., 1963, v. 7, p. 337-369.

17. Фукс Л. Бесконечные абелевы группы, т. 2. М.: Мир, 1977, 416 с.

18. Heyer H. Probability Measures on Locally Compact Groups. Berlin-Heidelberg-New York: Springer-Verlag, 1977, 531 p. (Ergeb. Math. Grenzgeb., v. 94.)

19. Ронкин Л. И. Введение в теорию целых функций многих переменных. М.: Наука, 1971, $432 \mathrm{c.}$

20. Фельдман Г. М. О гауссовских распределениях на локально компактных абелевых группах. - Теория вероятн. и ее примен., 1978, т. 23, в. 3, с. 548-563.

Поступила в редакцию 3.XII. 2010

Исправленный вариант 